\title{
In-Gap Spin Excitations and Finite Triplet Lifetimes in the Dilute Singlet Ground State System $\mathrm{SrCu}_{2-x} \mathrm{Mg}_{x}\left(\mathrm{BO}_{3}\right)_{2}$
}

\author{
S. Haravifard, ${ }^{1}$ S.R. Dunsiger, ${ }^{1}$ S. El Shawish,${ }^{3}$ B.D. Gaulin, ${ }^{1,2}$ H.A. Dabkowska, ${ }^{1}$ M.T.F. Telling, ${ }^{4}$ and J. Bonča ${ }^{3,5}$ \\ ${ }^{1}$ Department of Physics and Astronomy, McMaster University, Hamilton, Ontario, L8S 4M1, Canada \\ ${ }^{2}$ Canadian Institute for Advanced Research, 180 Dundas St. W., Toronto, Ontario, M5G 1Z8, Canada \\ ${ }^{3}$ J. Stefan Institute, SI-1000 Ljubljana, Slovenia \\ ${ }^{4}$ Rutherford Appleton Laboratory, ISIS Pulsed Neutron Facility, Chilton, Didcot, Oxon OX110QX, UK \\ ${ }^{5}$ Faculty of Mathematics and Physics, University of Ljubljana, SI-1000 Ljubljana, Slovenia
}

\begin{abstract}
High resolution neutron scattering measurements on a single crystal of $\mathrm{SrCu}_{2-x} \mathrm{Mg}_{x}\left(\mathrm{BO}_{3}\right)_{2}$ with $\mathrm{x} \sim 0.05$ reveal the presence of new spin excitations within the gap of this quasi-two dimensional, singlet ground state system. Application of a magnetic field induces Zeeman-split states associated with $\mathrm{S}=1 / 2$ unpaired spins which are antiferromagnetically correlated with the bulk singlet. Substantial broadening of both the one and two-triplet excitations in the doped single crystal is observed, as compared with pure $\mathrm{SrCu}_{2}\left(\mathrm{BO}_{3}\right)_{2}$. Theoretical calculations using a variational algorithm and a single quenched magnetic vacancy on an infinite lattice are shown to qualitatively account for these effects.

PACS numbers: $75.25 .+\mathrm{z}, 75.40 . \mathrm{Gb}, 75.40 .-\mathrm{s}$
\end{abstract}

Quasi-two dimensional quantum magnets which display collective singlet or spin gap behavior are very topical due to the novelty of their ground states 1 and their relation to high temperature superconductivity in the copper oxides. There are relatively few such materials, and crystal growth difficulties have further limited their study in single crystal form. $\mathrm{SrCu}_{2}\left(\mathrm{BO}_{3}\right)_{2}$ is established as a realization of the two dimensional Shastry Sutherland model ${ }^{2}$ for interacting $\mathrm{S}=1 / 2$ dimers ${ }^{3.4}$. It is comprised of well separated layers of $\mathrm{Cu}^{2+}, \mathrm{S}=1 / 2$ orthogonal dimers arranged on a square lattice. The material crystallizes into the tetragonal space group I $42 \mathrm{~m}$ with room temperature lattice parameters of $\mathrm{a}=8.995 \AA$, $\mathrm{c}=6.649 \AA^{5}$.

$\mathrm{SrCu}_{2}\left(\mathrm{BO}_{3}\right)_{2}$ has been well studied by an array of experimental techniques, which show it to possess a non-magnetic ground state. In particular earlier

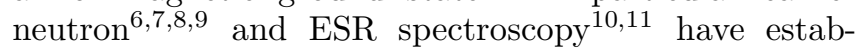
lished the leading terms in its microscopic Hamiltonian:

$$
\mathcal{H}=J \sum_{n n} \mathbf{S}_{\mathbf{i}} \cdot \mathbf{S}_{\mathbf{j}}+J^{\prime} \sum_{n n n} \mathbf{S}_{\mathbf{i}} \cdot \mathbf{S}_{\mathbf{j}}+g \mu_{B} \mathbf{H} \cdot \sum_{i} \mathbf{S}_{\mathbf{i}}
$$

where $\mathrm{J}$ is the exchange interaction within the dimers and $\mathrm{J}^{\prime}$ is the exchange interaction between $\mathrm{S}=1 / 2$ spins on neighboring dimers. Subleading DzyaloshinskiiMoriya interactions weakly split the three triplet modes even in zero applied magnetic field ${ }^{7.8 .9 .12}$.

Both the exchange interactions are antiferromagnetic and their ratio $\mathrm{x}=\frac{J^{\prime}}{J}$ has been estimated between 0.68 and 0.60 , with more recent refinements being smaller. Theoretically, such a quantum magnet is known to possess a singlet ground state so long as the ratio, $\mathrm{x}$, of inter to intra-dimer antiferromagnetic exchange is sufficiently small ${ }^{13}$. All of these estimates place $\mathrm{SrCu}_{2}\left(\mathrm{BO}_{3}\right)_{2}$ on the low side of the critical value of $\mathrm{x}$ at which a quan- tum phase transition occurs between a four sublattice Neel state and a collective singlet state.

In finite magnetic field, much interest has focused on a finite magnetization which develops at fields beyond $\sim$ $20 \mathrm{~T}$, wherein the lowest energy of the three triplet states has been driven to zero energy 4.14.15.16 $^{\text {. The magnetic }}$ field acts as a chemical potential for the triplet density within the quasi-two-dimensional planes. Magnetization plateaus ensue at higher fields, corresponding to Bose condensation of the triplets at certain densities.

While pure $\mathrm{SrCu}_{2}\left(\mathrm{BO}_{3}\right)_{2}$ has been well studied, there is little information on this quantum magnet in the presence of dopants, and no reports on doped single crystals. This problem is very interesting by analogy with the remarkable properties of doped quasitwo dimensional Mott insulators and high temperature superconductivity ${ }^{17}$. $\mathrm{SrCu}_{2}\left(\mathrm{BO}_{3}\right)_{2}$ is itself a Mott insulator and the theory of doped Mott insulators on the Shastry-Sutherland lattice shows the possibility of several different superconducting phases as a function of doping 18.19.20. Several doping studies of $\mathrm{SrCu}_{2}\left(\mathrm{BO}_{3}\right)_{2}$ have been reported on polycrystalline samples wherein $\mathrm{Al}, \mathrm{La}, \mathrm{Na}$, and $\mathrm{Y}$ substitute at the Sr site and $\mathrm{Mg}$ substitutes at the $\mathrm{Cu}$ site 21 .

In this Letter we report high resolution time-of-flight neutron scattering measurements on large single crystals of $\mathrm{SrCu}_{2-x} \mathrm{Mg}_{x}\left({ }^{11} \mathrm{BO}_{3}\right)_{2}$ and $\mathrm{SrCu}_{2}\left({ }^{11} \mathrm{BO}_{3}\right)_{2}$. These measurements show that doping of the magnetic $\mathrm{Cu}^{2+}$ site with non-magnetic, isoelectronic $\mathrm{Mg}^{2+}$ at the $2.5 \%$ level introduces new magnetic excitations into the singlet energy gap, and gives a finite lifetime to all three single triplet excitations, while also substantially broadening the two triplet bound state.

Two single crystal samples, $\mathrm{SrCu}_{2}\left({ }^{11} \mathrm{BO}_{3}\right)_{2}$ and $\mathrm{SrCu}_{2-x} \mathrm{Mg}_{x}\left({ }^{11} \mathrm{BO}_{3}\right)_{2}$, were grown by floating zone image furnace techniques at a rate of $0.2 \mathrm{~mm} /$ hour in an $\mathrm{O}_{2}$ 


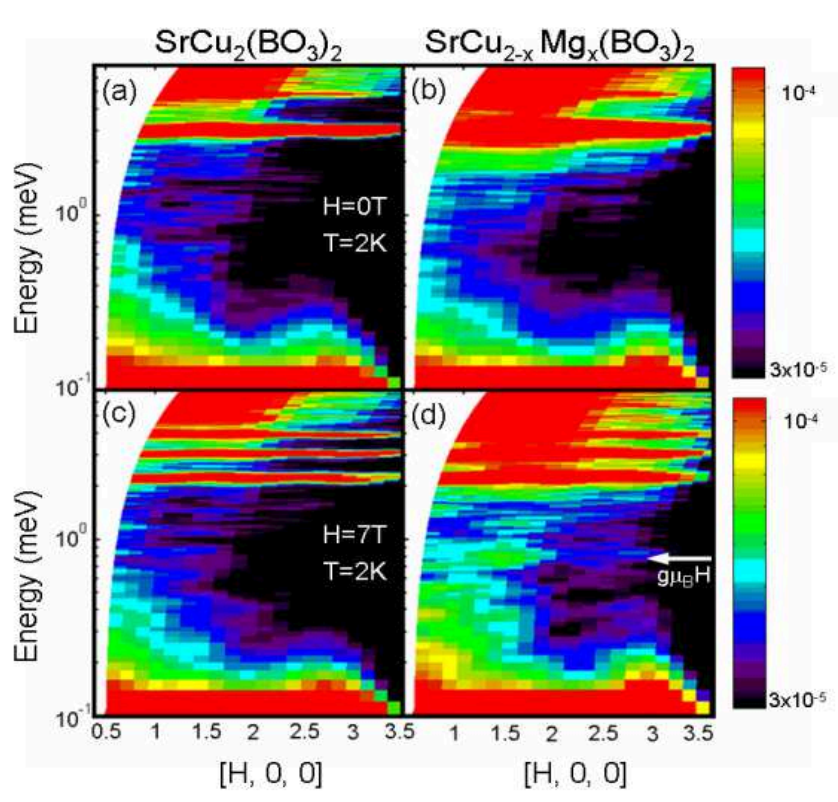

FIG. 1: (a) The two left hand panels show neutron scattering data from $\mathrm{SrCu}_{2}\left(\mathrm{BO}_{3}\right)_{2}$ at $\mathrm{T}=2 \mathrm{~K}$. The scattering has been integrated along $\mathrm{L}$ and we show data in a magnetic field of zero and $7 \mathrm{~T}$. The right hand panels show the same data for $\mathrm{SrCu}_{2-x} \mathrm{Mg}_{x}\left(\mathrm{BO}_{3}\right)_{2}$. The energy axis is on a logarithmic scale. The Zeeman-split $\mathrm{S}=1 / 2$ level in $\mathrm{SrCu}_{2-x} \mathrm{Mg}_{x}\left(\mathrm{BO}_{3}\right)_{2}$ in $\mathrm{H}=7 \mathrm{~T}$ at $\mathrm{g} \mu_{B} \mathrm{H}=0.8 \mathrm{meV}$ is indicated in panel $\mathrm{d}$ ).

atmosphere. The crystals were of almost identical cylindrical shape, with approximate dimensions of $4.5 \mathrm{~cm}$ in length by $0.6 \mathrm{~cm}$ in diameter. These samples were grown using ${ }^{11} B$, to avoid the high neutron absorption cross section of natural boron. The pure $\mathrm{SrCu}_{2}\left({ }^{11} \mathrm{BO}_{3}\right)_{2}$ single crystal was the same high quality single crystal studied previously ${ }^{9}$. Time-of-flight neutron scattering measurements were performed using the OSIRIS spectrometer ${ }^{22}$ at the ISIS Pulsed Neutron Source of the Rutherford Appleton Laboratory. OSIRIS is an indirect geometry time-of-flight spectrometer which employs an array of pyrolytic graphite monochromators to energy analyse the scattered neutron beam. The data was collected with the spectrometer configured to use the 004 analysing reflection afforded by pyrolytic graphite. In this configuration, only those neutrons with a scattered energy of $7.375 \mathrm{meV}$ are Bragg reflected towards the detector. The $[\mathrm{H}, 0, \mathrm{~L}]$ plane of both crystals was coincident with the horizontal scattering plane, and the samples were mounted in a $7 \mathrm{~T}$ vertical, $[0, \mathrm{~K}, 0]$, field magnet cryostat.

Figure 1 shows representative time-of-flight neutron scattering data, taken at $\mathrm{T}=2 \mathrm{~K}$ and $\mathrm{H}=0$ and $7 \mathrm{~T}$ for $\mathrm{SrCu}_{2}\left(\mathrm{BO}_{3}\right)_{2}$ (a and c) and for $\mathrm{SrCu}_{2-x} \mathrm{Mg}_{x}\left(\mathrm{BO}_{3}\right)_{2}$ (b and $\mathrm{d}$ ). This data was integrated along $\mathrm{L}$, in which direction the spin excitations show little dispersion ${ }^{9}$. Note the logarithmic energy and intensity scales, chosen to draw

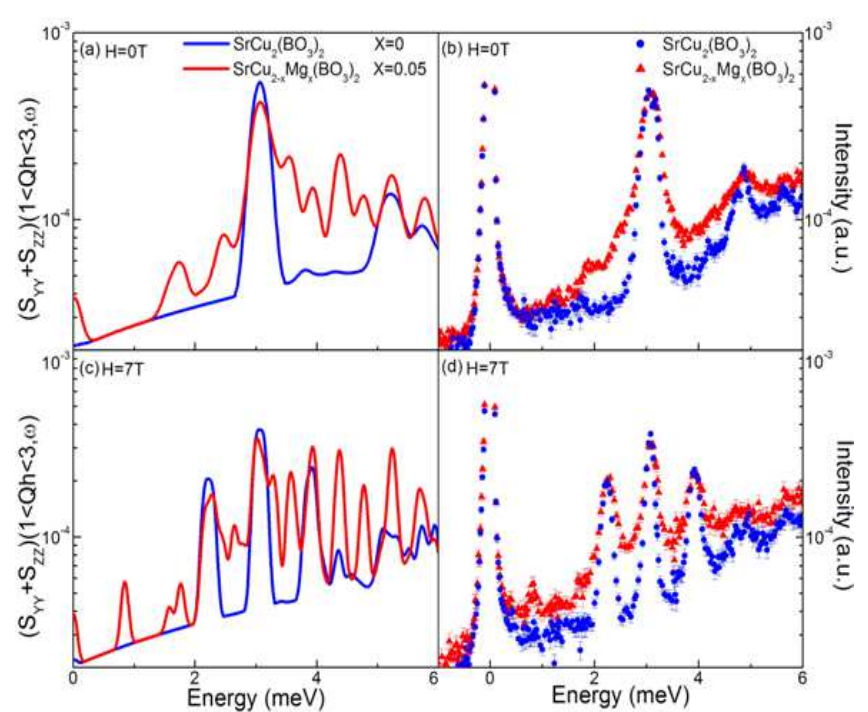

FIG. 2: The right hand panels show inelastic neutron scattering data for both $\mathrm{SrCu}_{2}\left(\mathrm{BO}_{3}\right)_{2}$ and $\mathrm{SrCu}_{2-x} \mathrm{Mg}_{x}\left(\mathrm{BO}_{3}\right)_{2}$ in $\mathrm{H}=0$ (b) and $\mathrm{H}=7 \mathrm{~T}(\mathrm{~d})$. This data has been integrated in $\mathbf{Q}$ between $\mathrm{H}=1$ and $\mathrm{H}=3$ and over all $\mathrm{L}$. The intensity is plotted on a logarithmic scale. Clearly the one and two triplet excitations are considerably broader in energy in $\mathrm{SrCu}_{2-x} \mathrm{Mg}_{x}\left(\mathrm{BO}_{3}\right)_{2}$ as compared with $\mathrm{SrCu}_{2}\left(\mathrm{BO}_{3}\right)_{2}$, and ingap states are introduced on doping. The left hand panels show the numerical calculation for $\mathrm{S}^{z z}(\mathbf{Q}, \omega)+\mathrm{S}^{y y}(\mathbf{Q}, \omega)$, using $\mathrm{J}=76.8 \mathrm{~K}$ and $\mathrm{J}^{\prime} / \mathrm{J}=0.62$, which accounts for many of the qualitative features observed in $\mathrm{SrCu}_{2-x} \mathrm{Mg}_{x}\left(\mathrm{BO}_{3}\right)_{2}$.

attention to the details of the in-gap excitations seen in $\mathrm{SrCu}_{2-x} \mathrm{Mg}_{x}\left(\mathrm{BO}_{3}\right)_{2}$. The splitting of the triplet excitations near $3 \mathrm{meV}$ on application of the $7 \mathrm{~T}$ magnetic field is clear. In finite field, weak dispersion of the triplet excitations as a function of wavevector $\mathrm{H}$ is seen, and this has been attributed to subleading terms in the spin Hamiltonian - terms other than those in Eq. 1. There are several qualitative features evident on examination of this data. The one triplet excitations show significantly greater breadth in energy in $\mathrm{SrCu}_{2-x} \mathrm{Mg}_{x}\left(\mathrm{BO}_{3}\right)_{2}$ than in $\mathrm{SrCu}_{2}\left(\mathrm{BO}_{3}\right)_{2}$. In addition, application of a $\mathrm{H}=7 \mathrm{~T}$ magnetic field gives rise to an inelastic peak at $\mathrm{g} \mu_{B} \mathrm{H}=0.8$ $\mathrm{meV}$ in $\mathrm{H}=7 \mathrm{~T}$, which is centered around $[\mathrm{H} \sim 1.4,0,0]$ in Q-space, but extends in wavevector $\mathrm{H}$ to almost $[\mathrm{H} \sim 3,0$, $0]$. Both of these features are discussed at length below.

Figures $2 \mathrm{~b}$ and $2 \mathrm{~d}$ show the same experimental data as in Fig. 1, now integrated in wavevector along $\mathrm{L}$ and also in $\mathrm{H}$ between $\mathrm{H}=1$ rlu and $\mathrm{H}=3 \mathrm{rlu}$, and plotted as a function of energy. The data in a magnetic field of 0 and $7 \mathrm{~T}$ is shown in Figs $2 \mathrm{~b}$ and $2 \mathrm{~d}$, respectively. The extra breadth in both the single triplet excitations and the two triplet bound states above it is clear. Broad inelastic peaks appear within the gap, which possess little magnetic field dependence, indicating a longitudinal nature. Sharp, field-induced inelastic scattering at $\hbar \omega \sim$ $0.8 \mathrm{meV}$ is also evident. 
Numerical calculations have also been carried out using a new variational approach ${ }^{23}$ to solve the model of a single quenched impurity on the two dimensional Shastry-Sutherland lattice. This method generates a variational space by successively applying the offdiagonal parts of the Hamiltonian, Eq. 1, on the starting approximation for the single impurity ground state, which consists of a free spin $1 / 2$ neighboring the impurity site, embedded within a dimer background. The resulting small spin polaron structure and exponential growth of the variational space with each iteration guarantee good convergence of the spin polaron ground state, as well as for the lowest energy excited states. Full details will be given separately ${ }^{23}$. For energies below $3 \mathrm{meV}$ the method provides accurate and converged results for both the longitudinal, $\mathrm{S}^{z z}(\mathbf{Q}, \omega)$, as well as the transverse, $\mathrm{S}^{y y}(\mathbf{Q}, \omega)$ components of the dynamical spin structure factor. These are compared directly to the neutron scattering experiments in Fig. 2.

Figures $2 \mathrm{a}$ and $2 \mathrm{c}$ show the calculated $\mathrm{S}^{z z}(\mathbf{Q}, \omega)+$ $\mathrm{S}^{y y}(\mathbf{Q}, \omega)$, integrated over the same range of wavevectors as the experimental data, and at magnetic fields of 0 (a) and $7 \mathrm{~T}$ (c). The comparison between theory and experiment is qualitatively good. The numerical results confirm that quenched magnetic vacancies induce in-gap states, substantial spectral weight below the zero field gap energy of $\sim 3 \mathrm{meV}$. A component of these in-gap states show little magnetic field dependence, and appear in the $\mathrm{S}^{z z}(\mathbf{Q}, \omega)$, longitudinal channel. The calculation also shows the $\mathrm{g} \mu_{B} \mathrm{H}$ transverse, $\mathrm{S}^{y y}(\mathbf{Q}, \omega)$, excitation in finite magnetic field. Furthermore, the calculation ${ }^{23}$ allows a determination of the spatial distribution of the spin polaron $\mathrm{S}=1 / 2$ degree of freedom and its low lying excited states, and these can guide the interpretation of the Q-dependence of the field-induced $\mathrm{g} \mu_{B} \mathrm{H}$ transverse spin excitation observed in the experiments.

Figure 3 shows the $\mathbf{Q}$ dependence of the scattering around $\mathrm{g} \mu_{B} \mathrm{H}=0.8 \mathrm{meV}$ in an applied magnetic field of 7 $\mathrm{T}$ in both $\mathrm{SrCu}_{2}\left(\mathrm{BO}_{3}\right)_{2}(\mathrm{a})$ and $\mathrm{SrCu}_{2-x} \mathrm{Mg}_{x}\left(\mathrm{BO}_{3}\right)_{2}(\mathrm{~b})$. This scattering is integrated in wavevector over $\mathrm{L}$, and in energy between $0.6 \mathrm{meV}<\hbar \omega<1 \mathrm{meV}$ and is shown in a magnetic field of both 0 and $7 \mathrm{~T}$. Figure $3 \mathrm{a}$ shows the absence of a magnetic field induced signal in $\mathrm{SrCu}_{2}\left(\mathrm{BO}_{3}\right)_{2}$ within this energy range. Figure $3 \mathrm{~b}$ shows a clear field induced signal in $\mathrm{SrCu}_{2-x} \mathrm{Mg}_{x}\left(\mathrm{BO}_{3}\right)_{2}$ which peaks at $[\mathrm{H} \sim 1.4,0,0]$, but extends out to almost $[\mathrm{H} \sim 3.0$, $0,0]$. This field induced scattering is attributed to Zeeman split $\mathrm{S}=1 / 2$ states associated with the $\mathrm{S}=1 / 2$ moment in a dimer whose partner site is occupied by a quenched, nonmagnetic $\mathrm{Mg}^{2+}$ ion. This field induced inelastic scattering is very similar to that associated with end states in Haldane spin chains, such as occur in $\mathrm{Y}_{2} \mathrm{BaNi}_{1-x} \mathrm{Mg}_{x} \mathrm{O}_{5}{ }^{24}$. In this case, quenched, nonmagnetic $\mathrm{Mg}^{2+}$ ions produce finite $\mathrm{S}=1$ magnetic chains. Spin 1/2 degrees of freedom arise at the end of finite chains of $\mathrm{S}=1$ magnetic moments, as one of the two ef-

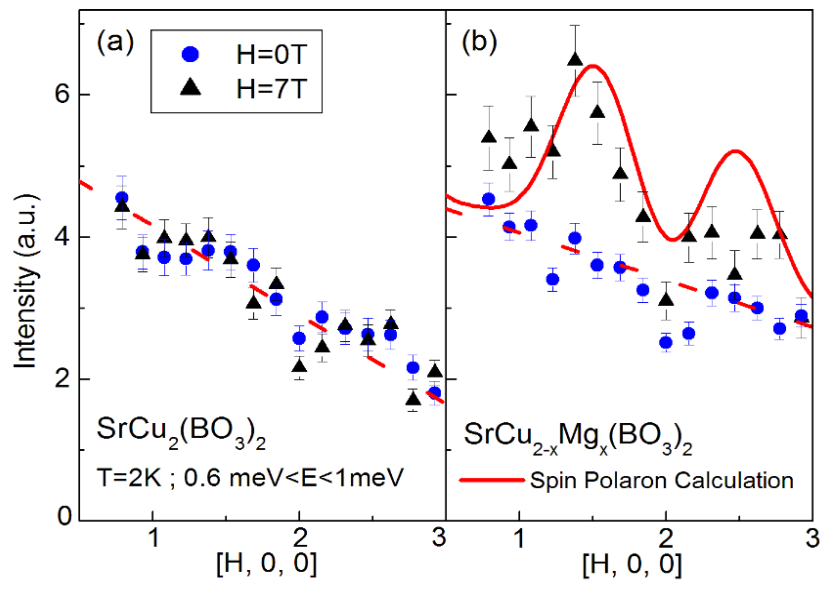

FIG. 3: Q-scans are shown at $\mathrm{T}=2 \mathrm{~K}$ for both $\mathrm{SrCu}_{2}\left(\mathrm{BO}_{3}\right)_{2}(\mathrm{a})$ and $\mathrm{SrCu}_{2-x} \mathrm{Mg}_{x}\left(\mathrm{BO}_{3}\right)_{2}$ (b) which integrate in energy between $0.6 \mathrm{meV}$ and $1 \mathrm{meV}$. This energy range captures the Zeeman energy $\mathrm{g} \mu_{B} \mathrm{H}=0.8 \mathrm{meV}$ appropriate to $\mathrm{g}=2$ and $\mathrm{H}=7 \mathrm{~T}$. (b) shows a comparison between the experimental Q-dependence of this scattering with the calculated form described in the text. The field-induced in-gap state in $\mathrm{SrCu}_{2-x} \mathrm{Mg}_{x}\left(\mathrm{BO}_{3}\right)_{2}$ is absent in $\mathrm{SrCu}_{2}\left(\mathrm{BO}_{3}\right)_{2}$.

fective $S=1 / 2$ degrees of freedom making up the $S=1$ moments lacks a partner with which to form a singlet. Such excitations occur at an energy of $\mathrm{g} \mu_{B} \mathrm{H}$ in finite field, and display a wavevector dependence which indicates antiferromagnetic correlations into the collective singlet of the chain segment.

The wavevector dependence of the magnetic fieldinduced spin excitation at $0.8 \mathrm{meV}$ can be attributed to the structure of the spin polaron ${ }^{23}$, whose ground state possesses strong antiferromagnetic correlations with neighboring dimers, transverse to the dimer containing the impurity site. To first non-trivial order in $J^{\prime} / J$, we obtain an analytical expression for the square of the matrix element for this transition:

$$
\begin{array}{r}
I^{ \pm} \propto \mid\left(a_{3}^{2}-a_{1}^{2} / 2\right) e^{\mathrm{i} \eta(\mathrm{H} \pm \mathrm{K})}+2 \sqrt{2} a_{1} a_{2} \times \\
\sin (\eta(\mathrm{H} \mp \mathrm{K})) \sin (\pi(\mathrm{H} \mp \mathrm{K}))-2 \sqrt{2} a_{2} a_{3} \times \\
\left.\cos (\eta(\mathrm{H} \mp \mathrm{K})) \cos (\pi(\mathrm{H} \mp \mathrm{K}))\right|^{2}
\end{array}
$$

where $a_{1}, a_{2}$, and $a_{3}$ are the weights of the polaron variational wavefunction computed for $J^{\prime} / J=0.62^{25} \cdot \eta=$ 0.72 accounts for microscopic distances in $\mathrm{SrCu}_{2}\left(\mathrm{BO}_{3}\right)_{2}$. The \pm sign in Eq. (2) distinguishes between the two nonequivalent impurity positions within the unit cell. Random doping therefore implies $I \propto I^{+}+I^{-}$, which we show, multiplied by the square of the magnetic form factor for $\mathrm{Cu}^{2+}$, in Fig. 3b along with measurements on Q-dependence of this scattering in $\mathrm{SrCu}_{2-x} \mathrm{Mg}_{x}\left(\mathrm{BO}_{3}\right)_{2}$. While the agreement between the calculation and experiment is not perfect, the calculation captures the general 


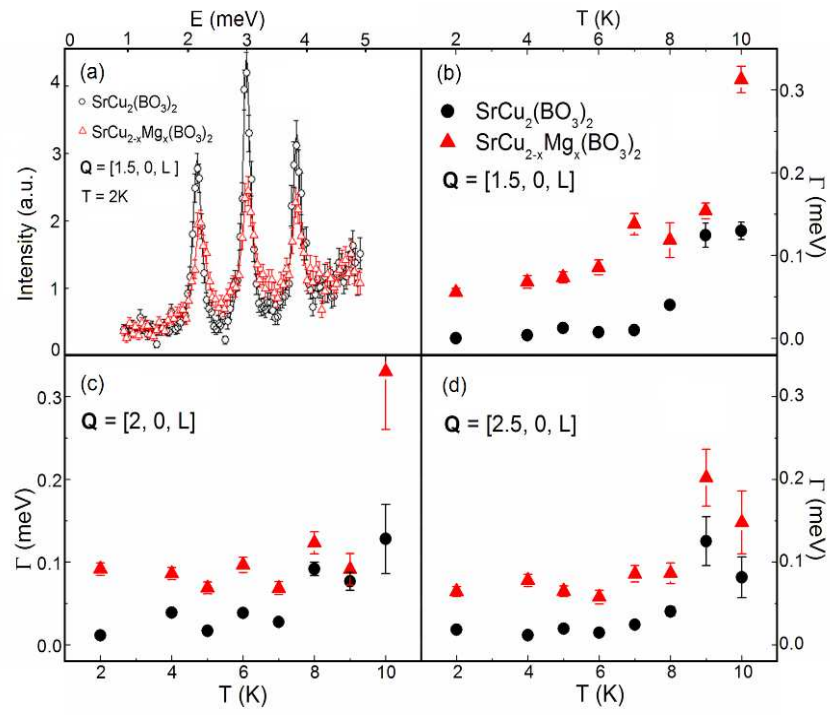

FIG. 4: (a) Cuts of the data simulating constant-Q scans at $(1.5,0, \mathrm{~L})$, integrated along $\mathrm{L}$, are shown for $\mathrm{SrCu}_{2}\left(\mathrm{BO}_{3}\right)_{2}$ and $\mathrm{SrCu}_{2-x} \mathrm{Mg}_{x}\left(\mathrm{BO}_{3}\right)_{2}$. Resolution convoluted fits to the data are shown as the solid lines and the description of the data is excellent. Such fits allow us to extract the triplet excitation lifetimes $(\Gamma)$, and which are shown in (b), (c), and d) for wavevectors $(1.5,0, \mathrm{~L}),(2,0, \mathrm{~L})$ and $(2.5,0, \mathrm{~L})$ respectively, as a function of temperature. Even at the lowest temperatures, we observe finite triplet lifetimes in $\mathrm{SrCu}_{2-x} \mathrm{Mg}_{x}\left(\mathrm{BO}_{3}\right)_{2}$.

\section{Q-dependence of the excitation.}

Figure 4a shows representative data with accompanying fits used to extract the lifetimes of the one triplet excitations as a function of doping and temperature in a magnetic field of $7 \mathrm{~T}$. This data is integrated in $\mathbf{Q}$ along $\mathrm{L}$, and over a narrow range in wavevector $\mathrm{H}$ around $\mathrm{H}=1.5$, and these cuts approximate constant- $\mathbf{Q}$ scans. Similar analysis was performed around wavevector $\mathrm{H}=2.0$ and 2.5 to look for variation in the one-triplet lifetimes as a function of $\mathbf{Q}$ along $[\mathrm{H}, 0,0]$. The data was fit to the sum of three identical damped harmonic oscillators (DHO):

$$
S(\mathbf{Q}, \omega, T)=\chi(\mathbf{Q}, T) \frac{1}{1-\exp \left(-\frac{\omega}{k T}\right)} \times
$$

$$
\left[\frac{4 \omega \Gamma_{\mathbf{Q}, T} / \pi}{\left(\omega^{2}-\Omega_{\mathbf{Q}, T}^{2}\right)^{2}+4 \omega^{2} \Gamma_{\mathbf{Q}, T}^{2}}\right]
$$

where $\chi(\mathbf{Q}, T)$ is the momentum-dependent susceptibility, while the second term takes into account detailed balance. The renormalized DHO frequency, $\Omega_{\mathbf{Q}}$, has contributions from the oscillation frequency, $\omega_{\mathbf{Q}, T}$, and the damping coefficient, $\Gamma_{\mathbf{Q}, T}$, and is given by: $\Omega_{\mathbf{Q}, T}^{2}=\omega_{\mathbf{Q}, T}^{2}+\Gamma_{\mathbf{Q}, T}^{2}$

Equation 3 was convoluted with an appropriate resolution function and fit to the data. The results are shown in Figs. 4b), c), and d), for wavevectors $\mathrm{H}=1.5,2.0$, and 2.5 respectively. The extracted lifetimes show little or no systematic variation with wavevector $\mathrm{H}$, but a finite one-triplet lifetime is observed in $\mathrm{SrCu}_{2-x} \mathrm{Mg}_{x}\left(\mathrm{BO}_{3}\right)_{2}$ at all temperatures, in contrast to $\mathrm{SrCu}_{2}\left(\mathrm{BO}_{3}\right)_{2}$, where the low temperature one-triplet lifetimes are very long, compared with the resolution of the spectrometer. In both $\mathrm{SrCu}_{2-x} \mathrm{Mg}_{x}\left(\mathrm{BO}_{3}\right)_{2}$ and $\mathrm{SrCu}_{2}\left(\mathrm{BO}_{3}\right)_{2}$, the thermal destruction of the collective singlet ground state near $\sim 10$ $\mathrm{K}$ is characterized by a rapid decrease in the one triplet lifetime $(1 / \Gamma)$ on warming, with little or no softening of the one triplet excitation energies.

As mentioned previously, the theoretical results for $\mathrm{S}^{z z}(\mathbf{Q}, \omega)+\mathrm{S}^{y y}(\mathbf{Q}, \omega)$ are not well converged for energies of $\sim 3 \mathrm{meV}$ and greater. Nonetheless, the additional broad spectral weight around the calculated one and two triplet energies seen in Fig 2a) and c) is consistent with finite triplet lifetimes in the presence of a single quenched magnetic vacancy.

To conclude, new inelastic neutron scattering measurements on $\mathrm{SrCu}_{2-x} \mathrm{Mg}_{x}\left(\mathrm{BO}_{3}\right)_{2}$ with $\mathrm{x} \sim 0.05$ show relatively broad and field-independent in-gap spin excitations as well as a magnetic field-induced excitation identified as a Zeeman-split, spin polaron state. The nonmagnetic quenched vacancies also give rise to finite and measurable lifetimes in the one and two triplet excitations.

We wish to acknowledge very helpful discussions with T.G. Perring and expert technical support from the ISIS User Group. This work was supported by NSERC of Canada, and the Slovenian Research Agency under contract PI-0044.
1 see, for example: E. Dagotto and T.M. Rice, Science, 271, 618 (1996); Dynamical Properties of Unconventional Magnetic Systems, edited by A.T. Skjeltorp and D. Sherrington, NATO ASI Series, Series E, Applied Sciences 349 (Kluwer Academic Publishers, Boston, 1998).

2 B.S. Shastry and B. Sutherland, Physica B\&C, 108B, 1069 (1981).

${ }^{3}$ S. Miyahara and K. Ueda, Phys. Rev. Lett., 82, 3701
(1999).

4 H. Kageyama et al, Phys. Rev. Lett., 82, 3168 (1999).

5 R.W. Smith and D.A. Keszler, J. Solid State Chem. 93, 430 (1991).

${ }^{6}$ H. Kageyama et al., Phys. Rev. Lett., 84, 5876 (2000).

7 O. Cepas et al., Phys. Rev. Lett., 87, 167205 (2001).

${ }^{8}$ K. Kakurai, in Quantum Properties of Low Dimensional Antiferromagnets, edited by A. Ajiro and J.P. Boucher 
(Kyushu University Press, Fukuoka, 2002).

9 B.D. Gaulin et al., Phys. Rev. Lett., 93, 267202 (2004).

10 A. Zorko, D. Arčon, H. van Tol, L.C. Brunel, and H. Kageyama, Phys. Rev. B, 69, 174420 (2004).

11 H. Nojiri, H. Kageyama, Y. Ueda, and M. Motokawa, J. Phys. Soc. Jap., 72, 3243 (2003).

12 S. El Shawish, J. Bonča, and I. Sega, Phys. Rev. B, 72, 184409 (2005).

13 M. Miyahara and K. Ueda, J. Phys. Condens. Matter 15, R327 (2003).

14 K. Kodama et al., Science 298, 395 (2002).

15 K. Onizuka et al., J. Phys. Soc. Jap., 69, 1016 (2000).

16 G.A. Jorge et al., Phys. Rev. B, 71, 092403 (2005).

17 See, for example: J. Orenstein and A.J. Millis, Science, 288, 468 (2000).

18 B.S. Shastry and B. Kumar, Prog. Theor. Phys. Suppl.
145, 1 (2002).

19 T. Kimura, K. Kuroki, R. Arita, and H. Aoki, Phys. Rev. B, 69, 054501 (2004).

20 C-H. Chung and Y.B. Kim, Phys. Rev. Lett., 93, 207004 (2004).

21 G.T. Liu, J.L. Luo, N.L. Wang, X.N. Jing, D. Jin, T. Xiang, and Z.H. Wu, Phys. Rev. B, 71, 014441 (2005).

22 M.T.F. Telling and K.H. Andersen, Phys. Chem. Chem. Phys., 7, 1255 (2005).

23 S. El Shawish and J. Bonča, cond-mat/0607753

24 M. Kenzelmann, G. Xu, I.A. Zaliznyak, C. Broholm, J.F. DiTusa, G. Aeppli, T. Ito, K. Oka, and H. Takagi, Phys. Rev. Lett., 90, 087202 (2003).

${ }^{25}$ For $J^{\prime} / J=0.62$, we obtain $a_{1}=0.914, a_{2}=0.234$, $a_{3}=0.165$. 